Sorda \& Sonora (1) 2018: 5 - 23

DOI: https://doi.org/10.18800/sordaysonora.201801.001

\title{
Propuesta de investigación para el estudio sobre la adquisición de clasificadores en Lengua de Señas Peruana como segunda lengua
}

Research proposal for the study of the acquisition of classifiers in Peruvian Sign Language as a second language

\author{
Mateo C. Marino \\ Pontificia Universidad Católica del Perú \\ marino.mateo@pucp.edu.pe
}

\section{RESUMEN}

El presente documento ofrece una propuesta esquemática de investigación acerca de la adquisición de los clasificadores en Lengua de Señas Peruana como segunda lengua por parte de niños y niñas oyentes. Para tal propósito, se propone en el proyecto analizar a cuarenta niños y niñas oyentes en contextos en los que se emplean la Lengua de Señas Peruana. El objetivo del estudio consistirá en dar cuenta del procesamiento de adquisición de los clasificadores verbales en la Lengua de Señas Peruana en niños y niñas oyentes. Se plantea como hipótesis tentativa que la secuencia de desarrollo de los clasificadores verbales en Lengua de Señas Peruana es la adquisición de (1) clasificadores verbales de un argumento y (2) clasificadores verbales de dos argumentos. Se propone esta secuencia debido al mayor grado de complejidad morfológica y configuración espacial que presenta (2).

Palabras clave: lengua de señas peruana, adquisición de segunda lengua, clasificadores, comunidad Sorda 


\section{AbStract}

The present paper aims to provide a schematic proposal of research on the acquisition of classifiers in the Peruvian Sign Language as a second language by hearing children. For this purpose, it is proposed to analyze in the project forty hearing children in contexts in which the Peruvian Sign Language is used. The objective of the study is to account for the processing of the acquisition of verbal classifiers in the Peruvian Sign Language in hearing children. These are hypothetical hypotheses that have the sequence of development of the verbal classifiers in Peruvian Sign Language in the acquisition of (1) verbal classifiers of an argument and (2) verbal classifiers of two arguments. This sequence is proposed due to the greater degree of morphological and spatial complexity that it presents (2).

Keywords: Peruvian Sign Language, acquisition of second language, classifiers, Deaf community 


\section{Introducción}

Una comunidad Sorda ${ }^{1}$ refiere a las personas que, a causa de la pérdida parcial o total de la audición, han desarrollado otros medios de organización y expresión del pensamiento por medio de la modalidad gestual-visual; con ello, se crea una lengua enteramente capaz de expresar todo rasgo de pensamiento humano. A esto se le llamará Lengua de Señas (LLSS). La principal dificultad de estas personas Sordas no es la sordera en sí misma, sino la incomprensión de la comunidad oyente con la cual conviven. Posiblemente, este obstáculo se debe a que los oyentes no comprenden que esta lengua, siendo un medio de comunicación, es una vía por la cual el Sordo accede a la educación, a los aspectos culturales y a la sociedad en general. Además, en el Perú, no existen estudios profundizados desde disciplinas científicas que investiguen a nuestra comunidad Sorda. Las investigaciones sobre Lengua de Señas Peruana (LSP) son muy escasas (Parks y Parks 2009, entre otros) y, por ello, no se conocen muchas de las características demográficas, culturales o lingüísticas de nuestra comunidad Sorda peruana.

En el área de la Lingüística, la morfología de la LSP es reconocida, ya que tiene un conjunto de morfemas articulados simultáneamente. Esta complejidad se debe a que las LLSS pueden utilizar articuladores lingüísticos como las manos, el torso, la boca y otros rasgos faciales conjuntamente, como muchos estudios han demostrado (Liddell 1980; Sutton-Spence y Woll 1999; Boyes 2001; Sutton-Spence y Day 2001; Sandler y Lillo-Martín 2006, entre otros). En este contexto, se plantea una pregunta de investigación, a nuestro parecer, interesante para el área de adquisición de LLSS como segunda lengua (L2).

\section{1 Área de estudio: los clasificadores en LLSS}

La simultaneidad en LLSS puede dividirse en tres categorías (Vermeerbergen, Leeson y Crasborn 2): (1) simultaneidad manual, cuando se usan las dos manos como canales autónomos paralelos, es decir, cuando una mano codifica

1 Se escribe con mayúscula siguiendo la propuesta de Erting y Woodward (1979), quienes diferencian el término "Sordo" para quien se sienta miembro de una comunidad Sorda, mientras que el término "sordo" designa la condición meramente audiológica. 
señas distintas de las de la otra mano; (2) simultaneidad que emplea un canal oral conjuntamente con uno manual, como el caso del "mouthing"2; y (3) uso simultáneo de otros articuladores no manuales distinto de la boca, por ejemplo, el torso o la mirada, entre otros. La presente propuesta de investigación se concentrará en la primera categoría. En ella, se encuentra la construcción de los clasificadores en LLSS. Esta es un campo ideal que permite comprender la simultaneidad en la gramática de LLSS (Tang, Sze y Lam 285). Los clasificadores son un tipo de construcción gramatical presente en lenguas orales y en LLSS. Para una definición general, Zwitserlood nos menciona lo siguiente acerca de los clasificadores: "Típicamente, estos elementos son morfemas que denotan una característica saliente de una entidad, por ejemplo, la característica de ser humano, ser un animal o tener una forma particular”, (1). Por citar un caso concreto, en la lengua oral $\mathrm{Kumiai}^{3}$, se manifiestan clasificadores verbales, es decir, elementos verbales que modifican al verbo de acuerdo a las características de los sujetos o de la acción.

Cuadro 1: clasificadores verbales en Kumiai

\begin{tabular}{|l|l|l|}
\hline Clase I & $\mathrm{a}^{\prime}$ & Objeto largo \\
\hline Clase II & $\mathrm{tu}$ & Objeto redondo pequeño \\
\hline Clase III & $\mathrm{c}^{\prime}$ & Grupo de objetos \\
\hline
\end{tabular}

En esta lengua, se puede agregar cada clase de clasificador verbal al verbo $x i$ 'arrastrar' para añadirle un significado adicional: $a^{\prime}$ xi 'arrastrar un objeto largo', tuxi 'arrastrar un objeto redondo pequeño' y c'xi 'arrastrar un grupo de objetos'. Así mismo, Zwitserlood nos menciona que los clasificadores, en las LLSS, presentan construcciones similares: “consisten en una configuración manual particular en señas, configuraciones manuales que denotan caracte-

2 Para mayor detalle, consúltese a Sandler (2009) quien propuso cuatro categorías de los usos de la boca en Lenguas de Señas.

3 Para mayor detalle de esta lengua amerindia consúltese a Pritzker (2000). 
rísticas de los referentes nominales", (3). Para ello, se usan ambas manos: la mano dominante (parte activa de la seña) y la mano no dominante (parte complementaria de la seña). A modo de ejemplo, se presenta la siguiente oración: "el gato camina sobre la casa" en Lengua de Señas Española (LSE) (véase Imagen $\left.1^{4}\right)$.

Imagen 1: la oración "el gato camina sobre la casa" en LSE

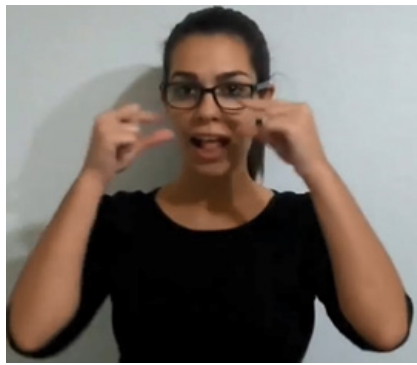

seña "GATO"

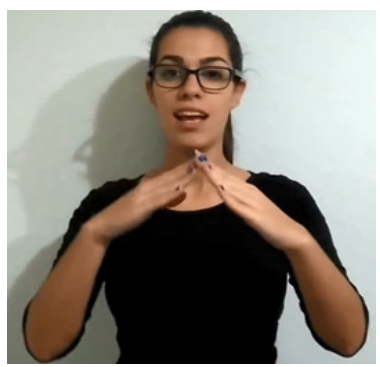

(iii) seña "CASA"

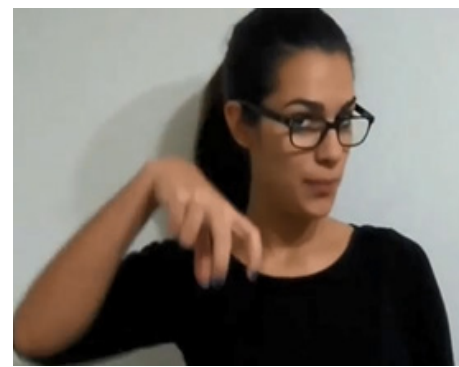

(ii) seña "CAMINAR"

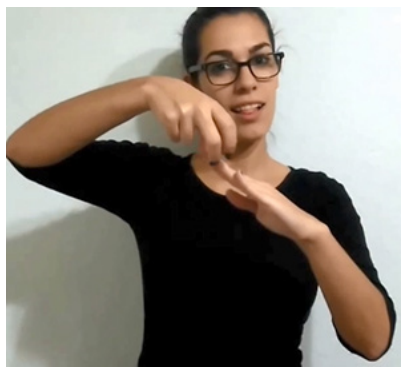

(iv) simultaneidad

En la Imagen 1, se aprecia que primero se signa la seña "GATO” con ambas manos. Luego, se usa la mano dominante para la seña "CAMINAR" y, de este modo, se ubica al sujeto ligado a la acción al lado derecho del espacio. Después, se signa la seña "CASA" con ambas manos. Por último, se puede apreciar que la mano no dominante mantiene la seña "CASA" a lado izquierdo del espacio y

4 Este ejemplo se ha tomado de un tutorial de Youtube sobre clasificadores en LSE. Se puede ubicar desde el segundo 0.20 hasta el segundo 0.30 por medio del siguiente enlace: https:// www.youtube.com/watch?v=nOgAr_2HKjA 
con ello permite que la mano dominante, ligada al sujeto y a la acción, pueda marcar la dirección del movimiento del verbo "caminar" para así terminar de construir la oración "el gato camina sobre la casa". En este sentido, la construcción simultánea en LLSS puede realizarse por medio de una seña léxica con un clasificador, como en (iv).

Dicho esto, nuestra propuesta plantea profundizar el procesamiento de la adquisición de los clasificadores verbales en la LSP. Este proceso es complejo, pues se basa en que la forma de la mano codifica una instrucción de procesamiento para ayudar al destinatario a escoger el referente a través de la inferencia y, una vez que se entienda al elemento referido, la forma de la mano puede desglosarse aún más para representar diferentes partes del mismo objeto aplicando el concepto de la forma de la mano. En este sentido, la construcción de los clasificadores es un tipo de expresión referencial.

\subsection{Pregunta principal sobre adquisición de LSP como L2}

Por ser una lengua de carácter gestual-visual, la LSP utiliza dimensiones de espacio y movimiento para transmitir información. Estos medios de organización proporcionan una fuente potencial para la simultaneidad de unidades léxicas o morfológicas. Las construcciones de los clasificadores verbales son ejemplos oportunos que demuestran la complejidad de esta simultaneidad en la gramática de LLSS, puesto que presentan una naturaleza principalmente icónica y están ligados al uso de verbos de movimiento o lugar, indicando el camino y la dirección del movimiento o el lugar del nombre al que se refieren. En este campo, algunos autores (Supalla 1990; Tang 2001, entre otros) se han interesado por estudiar las propiedades gramaticales de la mano y los componentes del movimiento. Por ello, el interés central es responder a la siguiente pregunta: ¿cómo adquieren los niños y las niñas oyentes la construcción de los clasificadores verbales en LSP como L2? Autores como Schick (1990) y Slobin (2003), con respecto a la Lengua de Señas Americana, mostraron que ciertas construcciones de clasificadores verbales no se adquirieron de manera uniforme o analógica; es decir, algunos niños Sordos presentaron secuencias diferentes de desarrollo y errores en la forma de las manos, el movimiento y la localización en el espacio en el proceso de adquisición. Siguiendo estos estudios, ahora sería interesante profundizar, desde un contexto diferente, la 
adquisición de estos clasificadores verbales en el procesamiento de aprendizaje de la LSP por parte de niños y niñas oyentes.

\section{Antecedentes}

La presente propuesta de investigación se basa, por un lado, en los trabajos de Tang, Sze y Lam (2007). Estos autores descubrieron que (1) los niños Sordos de la Lengua de Señas de Hong Kong adquieren los clasificadores verbales de predicados que involucran un argumento antes que los que implican dos, porque los predicados de dos argumentos reflejan un mayor grado de complejidad morfológica y, así, requieren de una configuración espacial más elaborada por los niños. Además, (2) estos niños Sordos se pueden dar cuenta de que algunas formas de las manos están asociadas con clasificadores nominales y otros, con clasificadores verbales específicos. Con relación a este estudio, la propuesta de este trabajo es describir cómo es el proceso de adquisición de los clasificadores verbales en LSP como L2 en un estudio con niños y niñas oyentes.

Por otro lado, nos sirve el inventario preliminar ${ }^{5}$ de Madrid (2017) de los clasificadores de LSP. En este, el autor nos brinda, por lo menos, 11 tipos de configuraciones manuales, de los cuales 6 se utilizan para referir directamente a los objetos (entity classifiers) y 7 se usan para referir indirectamente a los objetos (handling classifiers), los cuales se resumen en el Cuadro 2:

- Bípedo: representa entidades humanas (o con forma humana); así mismo, aparece representando a animales.

- Mano completa: suele aparecer cuando se hace referencia a objetos planos (puertas, periódicos, hojas de papel, entre otros), aunque también para aquellos que sean considerados como alargados.

- Índice: representa por lo general a entidades (humanos, pulpos, muñecos, cuchillos, entre otros).

- Vehículos: se le emplea para representar directamente a vehículos.

5 Tal proyecto de investigación es la realización de la tesis de Licenciatura del autor Madrid (2017), cuyos resultados iniciales se expusieron en la ponencia que se puede ubicar del minuto 44:40 al minuto 1:19:20 mediante el siguiente enlace de Youtube: https://educast.pucp.edu.pe/video/9442/ii_coloquio_internacional_sobre_la_ lengua_de_senas_peruana_parte_o8 
- Esfera: representa a entidades esféricas.

- Hoja de papel: representa indirectamente a objetos planos o para representar la manipulación con algo de delicadeza, no necesariamente plano.

- Vaso: se usa para representar indirectamente objetos cilíndricos (vasos, tazas, botellas, envases, entre otros).

- Mano cerrada: en las narraciones en LSP, refiere para indicar una manipulación de entidades realizada con fuerza.

- Asa: se utiliza para representar indirectamente a tazas (sujetadas por el asa) sartenes, cucharones, cepillos, entre otros.

- Punto del dedo: se utiliza para representar directamente a entidades de forma genérica.

- Índice y pulgar: se usa para representar indirectamente al hilo dental (entidad alargada y delgada).

Cuadro 2: Configuraciones manuales en LSP (Madrid 2017)

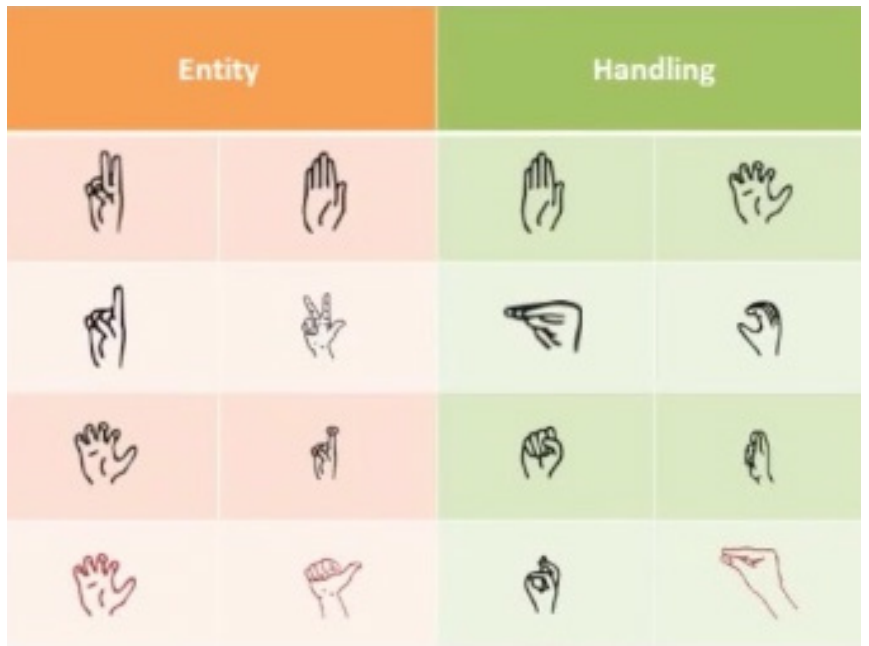

6 Este cuadro fue tomado de la ponencia de Madrid (2017) en el II Coloquio Internacional sobre Lengua de Señas Peruana, ubicado en el minuto 1:19:12 a través del siguiente enlace: https://educast.pucp.edu.pe/video/9442/ii_coloquio_internacional_sobre_ la_lengua_de_senas_peruana_parte_o8. 


\section{Propuesta}

\subsection{Pregunta específica}

¿Cuál es la secuencia de adquisición de los clasificadores verbales en la Lengua de Señas Peruana por parte de niños y niñas oyentes?

\subsection{Estructura lógica de la investigación}

Este estudio parte de los dos tipos de clasificadores en LSP, propuestos por Zwitserlood (2003): un grupo que refiere directamente a los objetos (entity classifiers) y otro que refiere indirectamente a los objetos, indicando su manipulación (handling classifiers). Estos dos tipos de clasificadores mantienen relación con los conceptos de figura, entidad movible cuyo camino, lugar u orientación se trata de determinar; y fondo, entidad estática con relación al marco de referencia, que determinará el camino, el lugar o la orientación de la figura. Nos interesa, en particular, los clasificadores verbales de predicados que involucran un argumento y los que implican dos argumentos.

Sobre la base de ello, se plantea como hipótesis tentativa que la secuencia de desarrollo de los clasificadores verbales en LSP es la adquisición de (1) clasificadores verbales de un argumento y (2) clasificadores verbales de dos argumentos. Se propone esta secuencia debido al mayor grado de complejidad morfológica y configuración espacial que presenta (2).

De confirmarse la hipótesis, se permitirá demostrar que el procesamiento de adquisición de los clasificadores se desarrolla siguiendo un patrón de complejidad icónico y espacial, al igual que la adquisición por parte de niños Sordos como lo proponen Tang, Sze y Lam (2007). Por el contrario, de no confirmarse, proporcionaría evidencia de que el aprendizaje de una lengua oral influiría en la adquisición de una lengua de señas como L2. Para que ello pueda darse, tendría que haber secuencias diferentes de desarrollo y errores en la forma de las manos, ya sea con el movimiento o la localización en el espacio en la realización de dicho clasificador. 


\section{Diseño}

El presente estudio se concentra en la forma de las manos para la realización de los clasificadores en LSP. Ante esto, se presenta un diseño de factores mixtos 2x3x2 identificables en tres variables: cuasi-independientes, independientes y dependientes.

- Variable entre sujetos: edad en dos niveles niños de 5 a 6 años y de 10 a 13 años [cuasi-independiente].

- Variable intra-sujeto: complejidad del clasificador verbal de un argumento frente al de dos argumentos [independiente].

- Variable intra-sujeto: características particulares como aciertos o errores en la forma de las manos ya sea con su movimiento o su localización en el espacio [dependiente].

\section{Métodos}

La metodología consistirá en tres sesiones, las cuales serán grabadas para elaborar una base de datos de trozos de discurso en LSP.

\subsection{Sujetos/Participantes}

Como número de sujetos se propone escoger veinte niños y veinte niñas oyentes, hablantes de castellano, en contextos en los que se emplean la LSP, preferiblemente, de padres o familiares Sordos. Todos ellos tendrán que ser residentes de Lima, aunque no necesariamente nacidos en esta ciudad. Las edades que se fluctúan serán de dos niveles: de 5 a 6 años y de 10 a 13 años.

\subsection{Procedimiento}

Se designarán 3 sesiones, las cuales se estima que durarán aproximadamente media hora con los niños de 5 a 6 años y una hora con los niños de 10 a 13 años. Además, se contará con un intérprete de LSP, quien traducirá los diálogos y narraciones a castellano. En todas las sesiones, se usará una cámara con micrófono incorporado, el cual enfocará al niño y registrará el sonido de las instrucciones y la traducción del intérprete. 
Como se precisó, las sesiones estarán divididas en tres: la primera sesión consistirá en (1) presentar un número de tarjetas con dibujos de uno o dos personajes realizando alguna acción para luego pedirles a los niños que traten de formar oraciones o historias cortas que luego serán narradas. Esta elicitación se sigue de Sardihnha (2011), quien elaboró tales tarjetas y las dispuso a disposición de todos como recurso online disponible mediante el enlace www. story-builder.ca. Esto contribuirá a apreciar cómo los niños describen eventos y estados, y ordenan estos en secuencias temporales. Así mismo, (2) se propone usar cortos animados sin diálogos, los cuales presenten una historia emocionante y variada en los que se manejen grados de iconicidad distinta. Dichos cortos tendrán que cumplir con los requisitos de concebir una historia compleja con muchas acciones y con conceptos abstractos. Los cortos escogidos posiblemente sean nominados al Oscar o a premios en festivales internacionales en los últimos años como French Roast (2010) o La Dama y la Muerte (2010)7. La segunda sesión consistirá en generar oraciones en las que se pedirá a los niños que conversen entre sí en LSP. Con el fin de estimular esta conversación, se les pedirá que cada uno le cuente al otro sobre las historias antes vistas de cortos animados. Estas historias se han pensado tomar de la serie Simon's Cat, cuyo artista gráfico es Simon Tofield. Como punto de partida, se empezará con estas historias: "Camouflage Challenge", "Cat vs Boxes" y "Bed Head”. El contenido de estos cortos animados favorece al objetivo de elicitar historias, cuyas tramas estén vinculadas al uso de verbos de movimiento y referencias locativas, pues las mascotas en las historias se mueven en un ambiente pequeño por diferentes puntos con respecto a su dueño. La última sesión (3) estará designada a la retroalimentación para el investigador; es decir, se aprovechará esta sesión para preguntar sobre las dudas de algunas partes de las sesiones anteriores. Todas estas grabaciones serán transcritas en ELAN para su análisis posterior. Este programa es el mejor software gratuito para la transcripción, anotación y codificación de archivos audiovisuales.

7 La duración de ambos cortometrajes animados es de 8 minutos aproximadamente y se pueden ubicar mediante los siguientes enlaces de youtube: (1) French Roast en https://www.youtube.com/watch?v=jbFhATUfuow y (2) La Dama y la Muerte en https://www.youtube.com/watch?v=8Zrw61WiBiQ 


\subsection{Relevancia del estudio}

En general, estudios enfocados en el análisis gramatical sobre LLSS en contacto con una lengua oral contribuyen a un mejor entendimiento sobre la condición sorda. Para este estudio en particular, proponer una secuencia de adquisición de los clasificadores en LSP como L2 permite demostrar su naturaleza icónica, así como la marcación de la relación espacial y de movimiento como recursos articulatorios en el proceso de aprendizaje. De esta manera, la presente propuesta aspira a demostrar que (1) los niños de dos grupos de edad diferenciados tienen manejos diferentes de los clasificadores verbales de predicados con un argumento frente a los de dos argumentos y (2) en cada grupo en particular puede haber más aciertos o errores en las realizaciones de la forma de las manos al momento de signar clasificadores en LSP. 


\section{Referencias bibliográficas}

Boyes Braem, Penny. "Functions of the mouthings in the signing of deaf early and late learners of Swiss German Sign Language (DSGS)" The hands are the head of the mouth: The mouth as articulator in sign languages, 2001, pp. 99-132.

Erting, Carol, y James Woodward. "Sign language and the deaf community a sociolinguistic profile." Discourse Processes vol. 2, no. 4, 1979 pp. 283-300. https://doi. org/10.1080/01638537909544469

Liddell, Scott K. American sign language syntax. Vol. 52. Mouton de Gruyter, 1980. Madrid, Rodrigo. Clasificadores en lengua de señas peruana (LSP). https://educast. pucp.edu.pe/video/9442/ii_coloquio_internacional_sobre_la_lengua_de_senas_peruana_parte_o8. Acceso 15 dic. 2017.

Parks, Elizabeth y Jason Parks. "Sociolinguistic survey report of the Deaf community of Peru”. Survey Report 19 del Summer Institute of Linguistics Electronic, 2009. SIL, www. sil.org/resources/publications/entry/9212. Acceso 15 dic. 2018.

Pichler, Deborah y Helen Koulidobrova. "Acquisition of sign language as a second language" The Oxford Handbook of Deaf Studies in Language. Marc Marschark, Patricia Elizabeth Spencer (editores). Oxford University Press, 2016.

Plaza-Pust, Carolina. Bilingualism and Drafness on language contact in the bilingual acquisition of sign language and written language. Mouton de Gruyter 2016, pp. 151155 .

Pritzker, Barry. A Native American encyclopedia: History, culture, and peoples. Oxford University Press on Demand, 2000.

Sandler, Wendy. The uniformity and diversity of language: Evidence from sign language. Lingua, vol. 120, no.12, 2010, pp. 2727-2732. https://doi.org/10.1016/j.lingua.2010.03.015

Sandler, Wendy y Diane Lillo-Martin. Sign language and linguistic universals. Cambridge University Press, 2006. https://doi.org/10.1017/CBO9781139163910

Sardinha, Katie. “Story-Builder,” Story Builder. http://www.story-builder.ca. Acceso 3 jul. 2017.

Schick, Brenda. "Classifier predicates in American Sign Language," International Journal of Sign Linguistics vol. 1, no.1, 1990, pp. 15-40.

Slobin, Dan I., et al. "A cognitive/functional perspective on the acquisition of "classifiers"," Perspectives on classifier constructions in sign languages, vol. 2, 2003, pp. 271296. 
Stokoe, William C. "Classification and description of sign languages," Current trends in linguistics, vol. 12, no. .pt 2, 1974, pp. 345-371.

Sutton-Spence, Rachel, y Linda Day. "Mouthings and mouth gestures in British Sign Language (BSL)," The hands are the head of the mouth: The mouth as articulator in sign languages, 2001, pp. 69-85.

Supalla, Ted. "Structure and acquisition of verbs of motion and location in American Sign Language”, University of California, San Diego, 1982.

Supalla, Ted. "Serial verbs of motion in ASL," Theoretical issues in sign language research, vol. 1, 1990, pp. 127-152.

Tang, Gladys. "Classifiers of Hong Kong Sign Language: a semantic universals perspective." Proceedings of the 12th North American Conference on Chinese Linguistics. San Diego State University, San Diego, CA, 2000, pp. 187-207.

Tang, Gladys, Felix Sze, y Scholastica Lam. "Acquisition of simultaneous constructions by deaf children of Hong Kong Sign Language," Amsterdam Studies in the Theory and History of Linguistic Science Series 4, vol. 281, 2007, pp. 283-316. https://doi. org/10.1075/cilt.281.13tan

Vermeerbergen, Myriam, Lorraine Leeson, and Onno Crasborn. "Simultaneity in signed languages: A string of sequentially organised issues," Amsterdam Studies in the Theory and History of Linguistic Science Series 4, vol. 281, 2007, p. 1. https://doi.org/10.1075/ cilt.281.01ver

Zwitserlood, Inge. "Classifying Hand Configurations in Nederlandse Gebarentaal (Sign Language of the Netherlands)”. PhD Dissertation, Utrecht University. Utrecht: LO, 2003. 


\section{Anexo 1}

Título del Protocolo: Adquisición de clasificadores en Lengua de Señas Peruana como segunda lengua

Investigador Principal: Marino Mateo Carrión (marino.mateo@pucp.edu.pe)

En esta forma de consentimiento, "usted" se refiere al padre, madre o tutor legal del niño sujeto de estudio. Esta forma no puede ser firmada por otro miembro de la familia del niño.

Le pedimos que lea el siguiente texto para que se informe sobre la naturaleza de esta investigación, y sobre lo que se espera de su hijo y de usted si participan en ella. El Comité de Ética para la Investigación con Seres Humanos y Animales de la Pontificia Universidad Católica del Perú requiere que le demos una forma de consentimiento por escrito. Si quiere participar, al firmar este consentimiento indicará que entiende de qué se trata esta investigación y que quiere participar en ella. También querrá decir que decidió participar de manera libre e informada.

\section{Introducción}

Le pedimos que participe voluntariamente en la investigación descrita abajo. Antes de aceptar participar en esta investigación, es importante que lea este texto. Por favor, pídale al equipo de investigación que le explique cualquier palabra o información que no entienda con claridad.

\section{¿Por qué se está haciendo este estudio?}

Esta investigación estudia la adquisición de las formas de la mano en uso simultáneo de la Lengua de Señas Peruana como Segunda Lengua por parte niños oyentes entre 5 a 6 años y 10 a 13 años. Aproximadamente, 40 niños participarán de este estudio en Lima. Le pedimos que participe en este estudio, porque su hijo es un niño pequeño que habla una lengua oral y que está en el proceso de aprendizaje de la Lengua de Señas Peruana. Si decide participar en esta investigación, la participación de su hijo durará aproximadamente media 
hora, si su hijo tiene entre 5 a 6 años, y una hora si su hijo tiene entre 10 a 13 años.

\section{¿Qué pasará durante la investigación?}

El investigador le pedirá que su hijo vea dibujos o cortos animados sin diálogo para que luego trate de narrarlos en Lengua de Señas Peruana. La sesión tendrá lugar en el lugar que usted prefiera. En esta investigación no hay respuestas correctas o incorrectas, así que a ningún niño le puede ir bien o mal. Estamos tratando de descubrir qué es lo más natural para los niños.

¿Cuáles son los riesgos o las molestias de la investigación?

Esta investigación no involucra más riesgo que aquellos asociados con actividades diarias.

\section{¿Hay beneficios por participar en esta investigación?}

No habrá beneficios directos para usted ni para su hijo por participar en esta investigación. Sin embargo, esta investigación nos ayudará a entender cómo se produce la adquisición de la forma de las manos en uso simultáneo en la Lengua de Señas Peruana como Segunda Lengua.

\section{¿Qué otras opciones hay?}

Tiene la opción de no participar en esta investigación. No sufrirá ninguna penalidad si decide no participar en esta investigación. Si después de empezar a participar, no quiere seguir por cualquier razón, la sesión se acaba. Puede parar cuando quiera.

\section{¿Cuáles son mis costos?}

No hay ningún costo directo. 


\section{¿Me pagarán por participar en esta investigación?}

No se le pagará por participar en esta investigación.

\section{¿A quién llamo si tengo preguntas o problemas relacionados con esta investigación?}

Puede preguntar lo que quiera al momento de recibir y leer este documento. $\mathrm{Si}$ tiene preguntas después, puede llamar a Marino Mateo al (---) o escribirle un mensaje electrónico a marino.mateo@pucp.edu.pe

\section{¿Qué pasa con la confidencialidad?}

Su participación en esta investigación es confidencial. Ninguna información se identificará por su nombre ni el de su hijo.

Grabaremos algunas sesiones de aplicación del cuestionario en audio y video para que podamos verificar su adecuada aplicación más tarde en nuestro laboratorio. Nuestras grabaciones son preservadas y estudiadas cuidadosamente a lo largo del tiempo.

Las grabaciones en audio y video, y los registros de información se guardan en computadoras protegidas a las que solo tienen acceso los investigadores autorizados. Los nombres y apellidos de su hijo y de usted serán borrados cuando cualquier dato sea reportado públicamente o en una publicación. Sus respuestas serán identificadas por un código y nunca por su nombre o apellido. Todas las grabaciones se marcan con un código para que no haya ninguna información de identidad personal visible en ellas. Sin embargo, ya que las cintas de video contienen su imagen, alguien que lo haya conocido (al momento de la grabación) podría identificarlo sin que nosotros revelemos su identidad. Además, durante las grabaciones, la investigadora lo llamará por su nombre de pila, pero su apellido nunca será mostrado públicamente en grabaciones de audio o video, o en ningún registro de información. Por lo tanto, abajo le pedimos autorización para usar las grabaciones, los datos de información, y los datos obtenidos de las grabaciones y el cuestionario de maneras específicas.

Los participantes en investigaciones sobre el lenguaje tienden a hablar con los investigadores y a conversar con ellos. Si los participantes nos dan infor- 
mación personal durante las sesiones de grabación más allá de la que pedimos en el cuestionario, esa información también será confidencial y la trataremos igual que el resto de los datos de esta investigación.

Por favor, infórmenos si en cualquier momento usted o su hijo (cuando sea mayor de edad) decide que no quiere que usemos sus datos en alguna forma; de lo contrario, asumiremos que tenemos su permiso para usarlos.

Nos gustaría muchísimo que participara en nuestra investigación. Si quiere darnos su consentimiento, por favor complete y firme el permiso siguiente.

Esta investigación está siendo llevada a cabo como trabajo final en el Seminario de Adquisición del Lenguaje en la Maestría en Lingüística de la Pontificia Universidad Católica del Perú.

\section{Documento de autorización}

Soy el padre, madre o tutor legal de (nombre del niño)

He leído este documento acerca de la investigación (o me ha sido leído). Sé que participar en esta investigación es voluntario y decidí participar en esta investigación. Sé que puedo parar de participar en esta investigación sin ninguna penalidad. Me darán una copia de este documento de consentimiento y puedo obtener información sobre los resultados de la investigación después si quiero.

Sección 1: Firmar esta sección quiere decir que acepta participar en el estudio. Firma del padre/tutor:

Nombre completo del padre/tutor:

Fecha de hoy:

Sección 2: En esta sección, le pedimos autorización adicional sobre el uso de sus datos en esta investigación. Esto es independiente de su participación en esta investigación. Por favor, marque todas las acciones que autorice y firme al final para mostrar que está de acuerdo. 
Top of form

\begin{tabular}{|l|l|}
\hline $\begin{array}{l}\text { Nos gustaría permitir que las investigado- } \\
\text { ras puedan... }\end{array}$ & Marque si está de acuerdo \\
\hline $\begin{array}{l}\text { Presentar el material de audio o video } \\
\text { de nuestras grabaciones en conferencias } \\
\text { científicas y en revistas académicas. }\end{array}$ & \\
\hline $\begin{array}{l}\text { Presentar el material de audio o video de } \\
\text { nuestras grabaciones en demostraciones } \\
\text { en salones de clase. }\end{array}$ & \\
\hline $\begin{array}{l}\text { Hacer los datos accesibles en el futuro a } \\
\text { otros investigadores fuera de Pontificia } \\
\text { Universidad Católica del Perú a través de } \\
\text { bases de datos de acceso restringido. Solo } \\
\text { investigadores que hayan recibido nuestra } \\
\text { autorización tendrán acceso a los datos. } \\
\text { Los datos nunca serán abiertos al público } \\
\text { en general. }\end{array}$ & \\
\hline
\end{tabular}

\section{Bottom of form}

Por favor, firme aquí si nos permite que compartamos sus datos de la(s) manera(s) descrita(s) arriba que usted ha marcado.

Firma del padre/tutor:

Si quiere recibir una copia de los resultados de esta investigación que reportemos, por favor, indique abajo su correo electrónico.

Forma de consentimiento entregada por:

Correo electrónico del padre/tutor: 